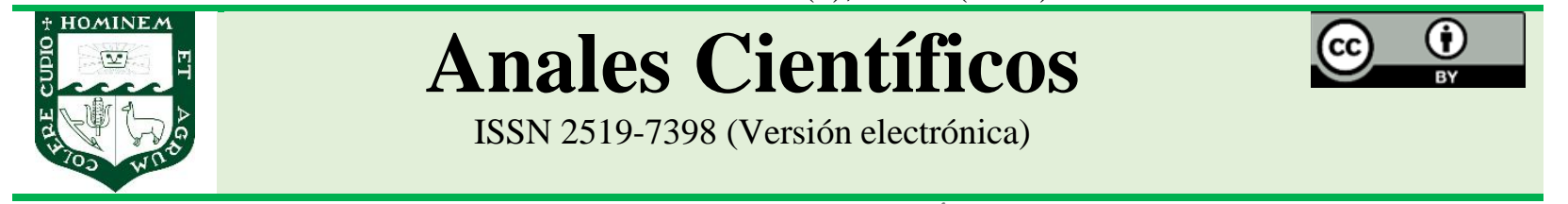

ARTÍCULO ORIGINAL - RESEARCH ARTICLE

http://dx.doi.org/10.21704/ac.v82i1.1744

\title{
MODELO DE ECUACIÓN ESTRUCTURAL EXPLICATIVO DEL DESEMPEÑO ESTUDIANTIL EN LA ASIGNATURA DE ESTADÍSTICA GENERAL EN LA UNALM
}

\section{Structural equation model explanatory of student performance in the subject of General Statistics at UNALM}

\author{
Rolando Salazar Vega* ${ }^{1}$; Fernando Rosas Villena ${ }^{1}[$ \\ ${ }^{1}$ Facultad de Economía y Planificación, Universidad Nacional Agraria La Molina, La Molina, 15024, Lima, Perú. \\ * E-mail: rsalazar@lamolina.edu.pe
}

Recibido: 21/05/2020; Aceptado: 27/06/2020; Publicado: 31/08/2021

\begin{abstract}
The purpose of the study is to measure the adjustment of an explanatory model of student performance in the subject of General Statistics at the La Molina National Agrarian University (UNALM) through the statistical technique of Structural Equation Models. The proposed model considers four factors: student performance, teacher performance, personal perception, and past performance. Two assessment instruments are applied in the study, first to measure personal perception, and the second, is to measure teacher performance. Cronbach's Alpha Reliability Coefficient is used to verify the reliability requirement and the Confirmatory Factor Analysis to verify the validity requirement of the assessment instruments. In both assessment instruments indicators obtain to confirm their reliability and validity. The fit of the proposed model was verified using the goodness of fit indicators, finding that the proposed model had a good fit. Said adjustment improved through a process of modifying the model through the inclusion of the relationship that indicates that personal perception depends on past performance. It verified the modified model that among the factors: past performance, teacher performance, and personal perception, the former is a predictor of student's performance. Also, in the modified model, where a new relationship includes, which was a past performance that explains the personal perception, this last relationship and the one that indicates that teacher performance explains personal perception were significant.
\end{abstract}

Keywords: Structural Equation Modeling; student performance; teacher performance; personal perception; past performance

\section{RESUMEN}

El estudio tiene como propósito medir el ajuste de un modelo explicativo del desempeño estudiantil en la asignatura de Estadística General en la Universidad Nacional Agraria La Molina (UNALM), mediante la técnica estadística de los Modelos de Ecuaciones Estructurales. El modelo propuesto considera cuatro factores: desempeño estudiantil, actuación docente, percepción personal y rendimiento pasado. En el estudio se aplican dos instrumentos de evaluación, el primero para medir la percepción personal y el segundo para medir la actuación docente. Se utiliza el Coeficiente de Confiabilidad de Alfa de Cronbach para verificar el requisito de confiabilidad y el Análisis Factorial Confirmatorio para verificar el requisito de validez, de los instrumentos de evaluación. En ambos 
Salazar, R., \& Rosas, F. (2021). Anales Científicos. 82(1), 83-91. DOI. 10.21704/ac.v82i1.1744

instrumentos de evaluación se obtuvieron indicadores que confirman su confiabilidad y validez. El ajuste de modelo propuesto se verificó mediante los indicadores de bondad de ajuste, encontrándose que el modelo propuesto presentaba un buen ajuste. Dicho ajuste fue mejorado mediante un proceso de modificación del modelo a través de la inclusión de la relación que indica que la percepción personal depende del rendimiento pasado. Se verificó en el modelo modificado que entre los factores rendimiento pasado, actuación docente y la percepción personal, el primero es predictor del desempeño estudiantil. También, en el modelo modificado donde se incluyó una nueva relación que es el rendimiento pasado explica la percepción personal, esta última relación y la que indica que la actuación docente explica la percepción personal resultaron significativas.

Palabras clave: Modelo de ecuaciones estructurales; desempeño estudiantil; actuación docente; percepción personal; rendimiento pasado

Forma de citar el artículo (Formato APA):

Salazar, R., \& Rosas, F. (2021). Modelo de Ecuación Estructural explicativo del desempeño estudiantil en la asignatura de Estadística General en la UNALM. Anales Científicos. 82(1), 83-91. http://dx.doi.org/10.21704/ac.v82i1.1744

Autor de correspondencia (*): Rolando Salazar Vega. Email: rsalazar@lamolina.edu.pe

(C) Los autores. Publicado por la Universidad Nacional Agraria La Molina.

This is an open access article under the CC BY

\section{INTRODUCCIÓN}

Las universidades tienen como funciones brindar una formación de calidad, desarrollar investigaciones y cumplir con su compromiso de responsabilidad social.

Las instituciones de enseñanza superior buscan alcanzar la calidad desarrollando sus procesos académicos y administrativos bajo una filosofía de mejora continua. Por eso es importante cuantificar la eficiencia de estos procesos relacionados, donde el desempeño estudiantil o rendimiento académico es un indicador en el proceso académico relacionado a medir el desempeño de un alumno.

Los Modelos de Ecuaciones Estructurales son una poderosa técnica del análisis multivariante que permite evaluar el ajuste de un modelo, probar la existencia de relaciones de dependencia entre factores, entre otras cosas. Guzmán (2012) hace un compendio de investigaciones en la que se verifica que el desempeño estudiantil se explica por modelos de ecuaciones estructurales en donde las variables se encuentran interrelacionadas. Guzmán (2012) y Flores et al. (2019) señalan que el rendimiento pasado es una variable importante que explica el desempeño estudiantil. Gargallo et al. (2009) indican "Hay algunas investigaciones que confirman la relación positiva significativa existente entre autoconcepto y rendimiento académico". Garbanzo
(2007) señala que los estudiantes buscan establecer con sus profesores una relación afectiva y de apoyo académico que les permita un mejor rendimiento académico. Según Muelas (2013) "Para aumentar el autoconcepto de un estudiante, la figura del docente va a ser clave". Cruz (2016) menciona que la metodología del docente, la empatía y el nivel académico previo influyen en el desempeño estudiantil.

Tomando en consideración los resultados de estas investigaciones, en el presente estudio se propone un modelo donde el desempeño estudiantil en la asignatura de Estadística General es explicado por tres factores: rendimiento pasado, percepción personal y actuación docente; además que el factor percepción personal se explica por el factor actuación docente. Entendiéndose que la percepción personal se refiere al autoconcepto.

Gargallo et al. (2009) mencionan que la percepción personal "se construye en interacción con el medio y va acompañada de connotaciones afectivas y evaluativas importantes" como el rendimiento pasado. Muelas (2013) indica que la relación entre la percepción personal y rendimiento académico se puede presentar en ambos sentidos. Por ello, como se verá en este trabajo, al modificar el modelo se incluirá la relación el rendimiento pasado como predictor de la percepción personal. 
Desde el punto de vista del criterio práctico, se justifica la investigación porque las áreas académicas de la UNALM relacionadas con el proceso enseñanza-aprendizaje contarán con un modelo explicativo del desempeño estudiantil en la asignatura de Estadística General con el cual podrán desarrollar estrategias para mejorar la calidad formativa de los alumnos.

La investigación tiene como objetivo medir el ajuste de un modelo propuesto de ecuación estructural que explica el desempeño estudiantil de los alumnos en la asignatura de Estadística General de la UNALM. Los objetivos específicos son : (1) verificar en el modelo de ecuación estructural los indicadores con valores aceptables, (2) verificar que el factor actuación docente se mide por las variables: organización del curso, dominio del curso, métodos y recursos pedagógicos, responsabilidades docentes, evaluación, motivación y relación con los alumnos, (3) verificar que el factor percepción personal se mide por las variables: autoconcepto académico, autoconcepto social, autoconcepto emocional, autoconcepto familiar y autoconcepto físico y (4) verificar que el factor percepción personal depende del factor actuación docente.

\section{MATERIALES Y MÉTODOS}

\section{Materiales}

Se utilizó el software R, versión 3.5.1

\section{Tipo de investigación}

La investigación es de tipo causal por las relaciones de dependencia entre determinados factores y correlacional por la asociación entre las variables o factores.

\section{Diseño de la investigación}

El diseño de la investigación es no experimental porque no existe manipulación de variables o factores y no hay control por la fuente de validez interna. Es de corte transversal porque los datos son obtenidos en un punto del tiempo determinado.

\section{Población y muestra}

La población está formada por 368 registros de los alumnos matriculados en la asignatura de Estadística General en el ciclo académico 2014-I. Considerando un error de estimación de 0,02 , un nivel de confianza de 0,99 y una probabilidad de éxito de 0,5 ; se obtuvo un tamaño de muestra de 338 registros el cual se mantuvo luego de realizar una imputación multivariada con el algoritmo MICE para los registros con datos ausentes.

\section{Metodología}

En el modelo propuesto de ecuación estructural que explica el desempeño estudiantil de los alumnos en la asignatura de Estadística General de la UNALM, se consideran las variables que se muestran en la Tabla 1.

Tabla 1. Especificación de las variables.

\begin{tabular}{|c|c|c|}
\hline Factores & Variables & Indicadores \\
\hline$\eta_{1}$ : Desempeño estudiantil & $\begin{array}{l}Y_{1} \text { : Nota final en la asignatura de } \\
\text { Estadística General }\end{array}$ & Promedio final \\
\hline \multirow[b]{6}{*}{$\xi_{1}:$ Actuación docente } & $\mathrm{X}_{1}$ : Organización del curso & Valor promedio de una escala de intervalo \\
\hline & $\mathrm{X}_{2}:$ Dominio del curso & Valor promedio de una escala de intervalo \\
\hline & $\mathrm{X}_{3}$ : Métodos y recursos pedagógicos & Valor promedio de una escala de intervalo \\
\hline & $\mathrm{X}_{4}$ : Responsabilidades docentes & Valor promedio de una escala de intervalo \\
\hline & $\mathrm{X}_{5}$ : Evaluación & Valor promedio de una escala de intervalo \\
\hline & X6: Motivación y relación con los alumnos & Valor promedio de una escala de intervalo \\
\hline$\xi_{2}:$ Rendimiento pasado & $\mathrm{X}_{7}$ : Promedio ponderado & Promedio ponderado acumulado \\
\hline \multirow{5}{*}{$\eta_{2}:$ Percepción personal } & $\mathrm{Y}_{2}$ : Autoconcepto académico & Valor promedio de una escala de intervalo \\
\hline & $\mathrm{Y}_{3}:$ Autoconcepto social & Valor promedio de una escala de intervalo \\
\hline & $\mathrm{Y}_{4}:$ Autoconcepto emocional & Valor promedio de una escala de intervalo \\
\hline & Y5: Autoconcepto familiar & Valor promedio de una escala de intervalo \\
\hline & Y6: Autoconcepto físico & Valor promedio de una escala de intervalo \\
\hline
\end{tabular}


Para procesar y analizar los datos, se consideraron tres etapas: (1) confiabilidad y validez, (2) validación del modelo y (3) propuesta de un nuevo modelo de ecuación estructural.

Etapa 1: Confiabilidad y validez

Aldás y Uriel (2017) señalan que para validar un instrumento de medida se debe: (1) realizar un Análisis Factorial Confirmatorio y (2) usar un conjunto de criterios para determinar la calidad del instrumento de medida, entendiéndose esto último como la confiabilidad.

Los requisitos de confiabilidad y validez para las encuestas de la percepción personal y actuación docente (ambas en una escala del 1 al 10) se verificaron con el coeficiente Alfa de Cronbach y el Análisis Factorial Confirmatorio respectivamente.

Etapa 2: Validación del modelo El modelo de ecuaciones estructurales propuesto se validó a partir de una adaptación de los pasos propuestos por Hair et al. (1999) y Schumacker y Lomax (2016): (1) especificación del modelo, (2) estimación del modelo, (3) identificación del modelo, (4) prueba del modelo e (5) interpretación y modificación del modelo.

Etapa 3: Propuesta de un nuevo modelo de ecuación estructural

Según Hair et al. (1999) y Véliz (2016), se puede realizar la re-especificación o modificación del modelo si es que se tiene justificación teórica para añadir o quitar relaciones en el modelo.

El modelo propuesto se modificó tomando en consideración los índices de modificación para encontrar las nuevas relaciones, las cuales deben tener un sustento teórico.

\section{RESULTADOS Y DISCUSIÓN}

Etapa 1: Confiabilidad y validez

En el requisito de confiabilidad de los instrumentos de evaluación de la percepción personal y de la actuación docente se tuvieron valores de Coeficientes de Confiabilidad Alfa de Cronbach de 0,80 y 0,939 respectivamente.
Se encontró que los instrumentos de evaluación de la percepción personal y actuación docente cumplen con el requisito de validez al encontrarse un porcentaje de variancia acumulada de $61,6 \%$ y $67,5 \%$ respectivamente.

Al verificarse que el Coeficiente de Confiabilidad Alfa de Cronbach tienen valores mayores o iguales al $80 \%$ y que los porcentajes de variancia acumulada son mayores al $60 \%$ en los instrumentos de evaluación de la percepción personal y de la actuación docente se puede concluir que dichos instrumentos tienen la calidad suficiente para poder estimar el modelo de ecuaciones estructurales respectivo.

Etapa 2: Validación del modelo

\section{Primer paso: Especificación del modelo}

Como resultado del primer paso se encuentra que el desempeño estudiantil depende del rendimiento pasado, de la percepción personal y de la actuación docente. También, se verifica que la percepción personal depende de la actuación docente.

En la Figura 1 se identifican los constructos exógenos: actuación docente y rendimiento pasado, y los endógenos: la percepción personal y el desempeño estudiantil. También se observan las relaciones entre los constructos que definen el modelo estructural.

Figura 1. Modelo estructural propuesto.

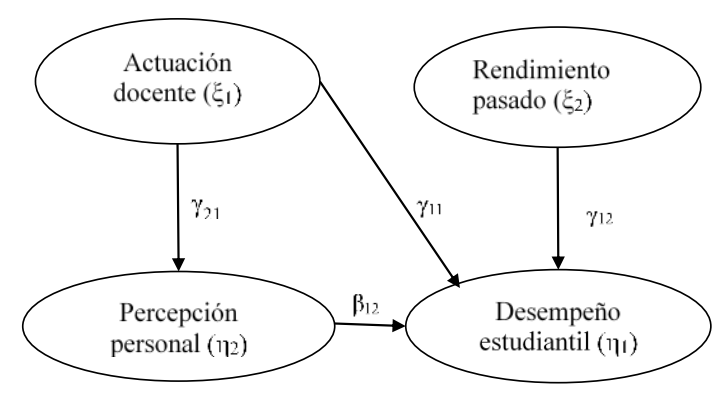

En la Figura 2 se identifican los constructos exógenos y endógenos con sus respectivos indicadores, y las relaciones entre los constructos. 


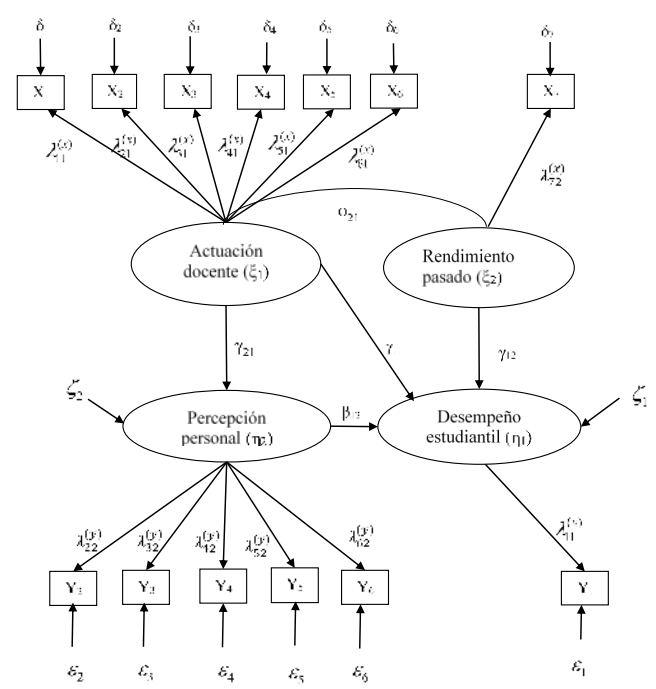

Figura 2. Modelo de ecuación estructural propuesto.

\section{Segundo paso: Estimación del modelo}

Mediante la aplicación de la prueba de HenzeZirkler se verificó que no se cumple el supuesto de normalidad multivariada de los datos, debido a que p-value $=0,000<\alpha=0,05$. Se comprobó la aleatoriedad de los datos ausentes en cada uno de los instrumentos de evaluación utilizando la prueba de Little's MCAR, concluyéndose que en ambos instrumentos son completamente aleatorios. Para estimar el modelo se utilizó la prueba escalada de Satorra-Bentler, imputándose 36 registros con datos ausentes en por lo menos un ítem, mediante el método de imputación multivariante con el algoritmo MICE, obteniéndose en total los 338 registros para hacer el análisis.

Tercer paso: Identificación del modelo

Al estimar los parámetros del modelo correctamente, se concluye que el modelo está identificado.

Cuarto paso: Prueba del modelo

Para determinar el ajuste del modelo no hay una única medida que, de un resultado definitivo, lo adecuado es usar en forma conjunta un grupo de medidas.

\section{Medidas de ajuste absoluto}

Hair et al. (1999) mencionan que "determinan el grado en que el modelo conjunto (modelos estructurales y de medida) predice la matriz de correlación o covariancia observada", se utilizaron los siguientes indicadores: Chi-Cuadrado robusto/grados de libertad, GFI, RMSEA robusto y SRMR robusto. El primer indicador fue de 3,02, valor considerado como medianamente aceptable por ser cercano a tres. El segundo indicador resultó 0,908 que por ser mayor a 0,90 indica buen ajuste del modelo. El tercer indicador resultó 0,085 , el cual estuvo ligeramente fuera del rango aceptable $<0,05$ 0,08> indicando entonces que el modelo es medianamente aceptable. Finalmente, el cuarto indicador fue 0,082 , el cuál por ser ligeramente mayor a 0,08 indicando que el modelo tiene un ajuste medianamente aceptable.

\section{Medidas de ajuste incremental}

Hair et al. (1999) mencionan que "compara el modelo propuesto con algún modelo de referencia, más a menudo referido como el modelo nulo", se utilizaron los siguientes indicadores: CFI robusto, TLI robusto y AGFI. El primer indicador fue de 0,912 el cual se encuentra dentro del rango aceptable $[0,90-0,95]$ indicando que el modelo tiene un ajuste aceptable. El segundo indicador resultó 0,889 por ser cercano al valor límite $(>0,90)$ indica que el modelo medianamente tiene un buen ajuste. El tercer indicador resultó 0,865 , el cual al ser cercano al valor límite $(\geq 0,90)$ indica que el modelo es medianamente aceptable.

\section{Medidas de ajuste de parsimonia}

Hair et al. (1999) mencionan que "relacionan la calidad del ajuste del modelo al número de coeficientes estimados exigidos para conseguir este nivel de ajuste", se utilizaron los siguientes indicadores: PGFI y PNFI. El primer indicador fue de 0,618 y el segundo indicador fue de 0,696 , los cuales son útiles para evaluar el nivel de complejidad del modelo. Estas medidas son útiles cuando se comparan modelos, por ello posteriormente estos resultados serán contrastados con los obtenidos luego de modificar el modelo.

Quinto paso: Interpretación y modificación del modelo 


\section{Modelo estructural}

Para determinar si las relaciones planteadas en el modelo estructural son significativas se compara el p-value con el nivel de significancia, obteniéndose los siguientes resultados. El rendimiento pasado explica el desempeño estudiantil ( $\mathrm{p}$-value $=0,000)$ y la actuación docente explica a la percepción personal ( $p$-value $=0,000$ ), y los factores percepción personal y la actuación docente no explican al desempeño estudiantil (p-value $=0,95$ y $\quad \mathrm{p}$ value $=0,136$ respectivamente) .

\section{Modelo de medida}

Para determinar si las relaciones planteadas en el modelo de medida son significativas se compara el p-value con el nivel de significancia, obteniéndose los siguientes resultados:

La actuación docente es explicada por las variables: organización del curso, dominio del curso, métodos y recursos pedagógicos, responsabilidades docentes, evaluación, motivación y relación con los alumnos (p-values $=0,000)$. La percepción personal es explicada por las variables: autoconcepto académico, autoconcepto social, autoconcepto emocional, autoconcepto familiar y autoconcepto físico $(\mathrm{p}$-values $=0,000)$

La modificación del modelo se realizó en la etapa 3.

Etapa 3: Propuesta de un nuevo modelo de ecuación estructural

En esta etapa se elimina o añade relaciones con el objetivo de tener un mejor ajuste o probar alguna relación teórica. Este proceso se debe realizar de forma bien pensada y fundamentada, por ello existen los índices de modificación para determinar las relaciones causales que pueden añadirse, el valor referencial para este índice es un valor mayor a 3,84. Con el software $\mathrm{R}$ se obtuvieron 41 relaciones que fueron evaluadas y finalmente se escogió la relación la percepción personal es explicada por el rendimiento pasado como aquella más adecuada y que tiene soporte teórico. Esta relación se incluyó en el modelo, se estimó el modelo, se observó que está identificado y se realizó la prueba del modelo, se verificó la significancia de la relación y resultó ser una relación importante, comprobándose que los indicadores de ajuste del modelo modificado son mejores que en el modelo propuesto (Figura 3).

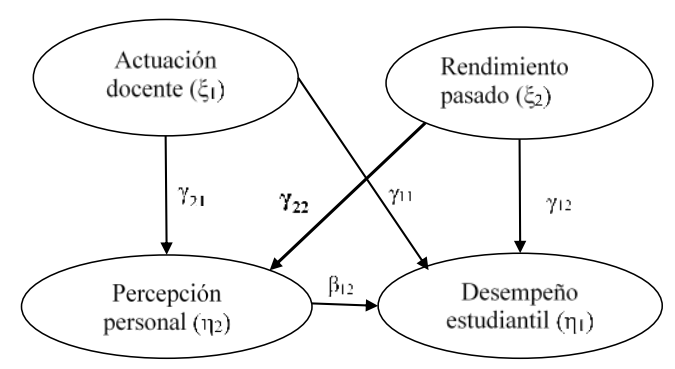

Figura 3. Modelo estructural modificado

\section{Análisis con el modelo modificado}

\section{Medidas de ajuste absoluto}

Se utilizaron los siguientes indicadores: ChiCuadrado robusto/grados de libertad, GFI, RMSEA robusto y SRMR robusto. El primer indicador fue de 2,716 valor considerado como aceptable al ser menor a tres. El segundo indicador resultó 0,912 que por ser mayor a 0,90 indica buen ajuste del modelo. El tercer indicador resultó 0,079 el cual está dentro del rango aceptable $\langle 0,05-0,08\rangle$ indicando entonces que el modelo es aceptable. Finalmente, el cuarto indicador fue de 0,073 , el cual es menor a 0,08 indicando que el modelo tiene un ajuste aceptable.

\section{Medidas de ajuste incremental}

Se utilizaron los siguientes indicadores: CFI robusto, TLI robusto y AGFI. El primer indicador fue de 0,927 el cual se encuentra dentro del rango aceptable $[0,90-0,95]$ indicando que el modelo tiene un ajuste aceptable. El segundo indicador resultó 0,906 , por ser mayor a 0,90 indica que el modelo tiene un buen ajuste. El tercer indicador resultó 0,869 , el cual al ser cercano al valor límite $(\geq 0,90)$ indica que el modelo es medianamente aceptable.

Medidas de ajuste de parsimonia

Se utilizaron los siguientes indicadores: PGFI y PNFI. El primer indicador fue de 0,611 y el segundo indicador fue de 0,696.

Evaluando el valor del PNFI en los modelos propuesto y modificado, la diferencia de ambos resulta cero, y considerando que un rango entre 0,06 y 0,09 indica diferencias significativas, entonces en 
base a este indicador se concluye que los modelos propuesto y modificado son semejantes en cuanto al nivel de ajuste.

Evaluando el valor del PGFI en los modelos propuesto y modificado, el modelo propuesto tiene un mayor valor de PGFI por eso se concluye que tiene mayor parsimonia.

\section{Modelo estructural}

Para determinar si las relaciones planteadas en el modelo estructural son significativas se compara el p-value con el nivel de significancia, obteniéndose los siguientes resultados:

El rendimiento pasado explica al desempeño estudiantil ( $p$-value $=0,000)$, la actuación docente explica a la percepción personal ( $p$-value $=0,000)$ y el rendimiento pasado explica a la percepción personal ( $\mathrm{p}$-value $=0,000$ ); y los factores percepción personal y la actuación docente no explican al desempeño estudiantil (p-value $=0,17$ y $p$ value $=0,26$ respectivamente).

\section{Modelo de medida}

Para determinar si las relaciones planteadas en el modelo de medida son significativas se compara el p-value con el nivel de significancia, obteniéndose los siguientes resultados:

La actuación docente es explicada por las variables: organización del curso, dominio del curso, métodos y recursos pedagógicos, responsabilidades docentes, evaluación, motivación y relación con los alumnos (p-values $=0,000)$. La percepción personal es explicada por las variables: autoconcepto académico, autoconcepto social, autoconcepto emocional, autoconcepto familiar y autoconcepto físico ( $p$-values $=0,00)$

\section{Análisis del modelo propuesto y modificado}

En el caso de los modelos propuesto y modificado, Guzmán (2012), Gargallo et al. (2009), Garbanzo (2007) y Cruz (2016) señalan que los factores rendimiento pasado, actuación docente y la percepción personal explican el desempeño estudiantil. Sin embargo, en la investigación cuando participan los tres en forma conjunta, solo el rendimiento pasado explica el desempeño estudiantil. Esto se explica porque gran parte de los alumnos que tienen buen desempeño estudiantil desde el inicio de su formación lo mantienen hasta el final. Se comprobó que el desempeño estudiantil no depende de la actuación docente. Esto se explica porque la asignatura de Estadística General forma parte de la componente de formación de estudios generales de la UNALM, en donde los cursos tienen características similares respecto a los programas, métodos y materiales utilizados, evaluación, metodología de enseñanza, entre otros. Se verificó que el desempeño estudiantil no depende de la percepción personal. Esto se sustenta porque la percepción personal está definida por 5 dimensiones (académico, social, emocional, familiar y físico) dentro de las cuales la dimensión académica tiene un impacto más significativo sobre el desempeño estudiantil.

Finalmente, se verificó que la percepción personal depende de la actuación docente. Muelas (2013) indica que la actuación docente es significativa para mejorar la percepción personal de los estudiantes. En el caso del modelo modificado, se encontró que el rendimiento pasado explica la percepción personal. Gargallo et al. (2009) y Muelas (2013) señalan los estudiantes forman su percepción personal en interacción con el medio en el cual se desenvuelven y que se ve influenciada por aspectos evaluativos como el rendimiento pasado.

En la Figura 4 que corresponde al modelo modificado, se muestra la interdependencia entre los constructos, la relaciones entre los constructos y sus indicadores, así como las cargas estandarizadas de dichas relaciones, siendo la relación más importante la que indica que el desempeño estudiantil se explica por el rendimiento pasado (carga explicativa de 0,56). También, se muestra que la percepción personal depende de la actuación docente y del rendimiento pasado. 


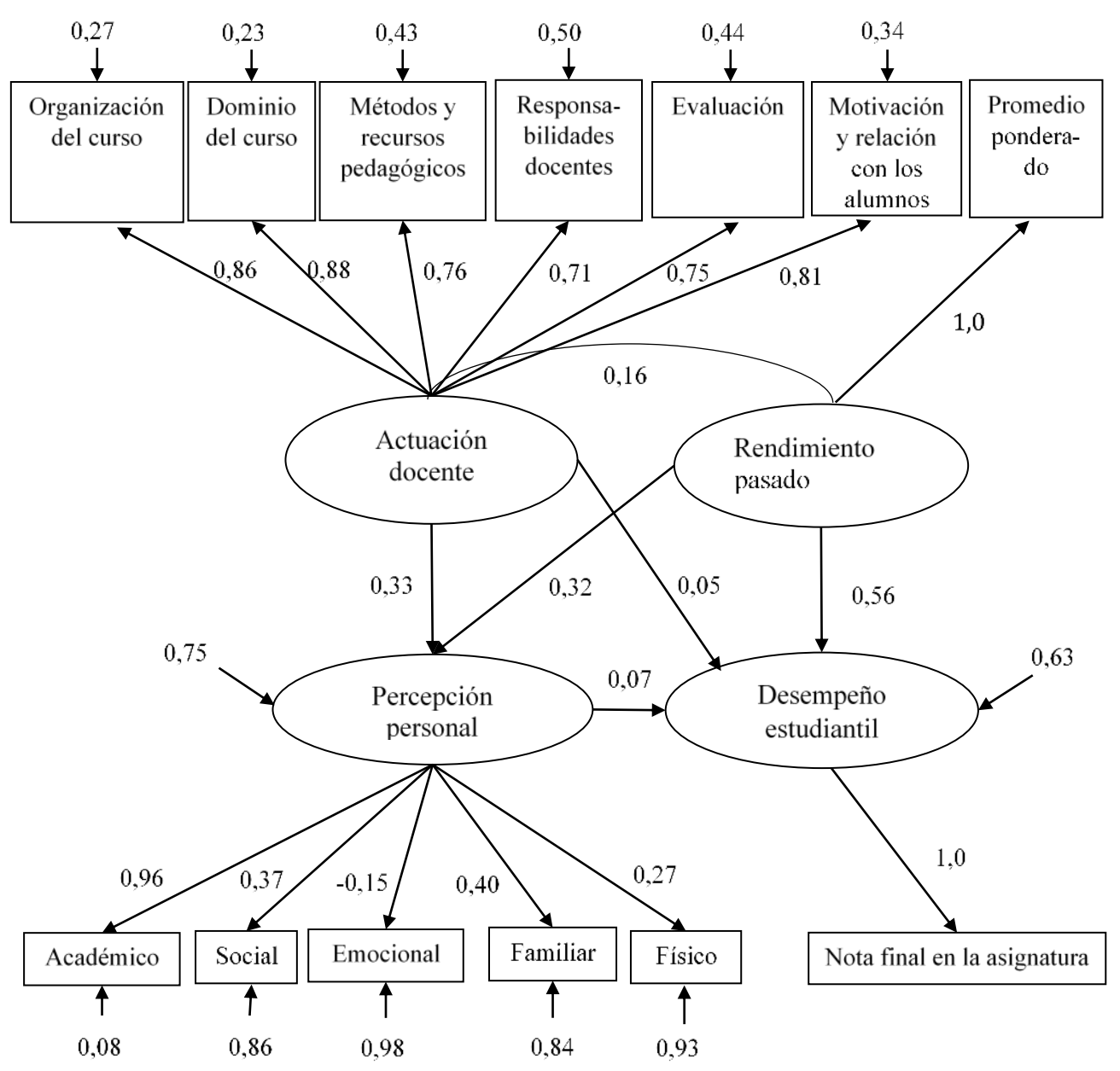

Figura 4. Modelo de ecuación estructural modificado estimado.

\section{CONCLUSIONES}

Se comprobó el buen ajuste del modelo propuesto de ecuación estructural que explica el desempeño estudiantil en la asignatura de Estadística General en el ciclo académico 2014-I. Se verifica que el rendimiento pasado explica el desempeño estudiantil, y que la actuación docente explica la percepción personal. Sin embargo, no se pudo comprobar que los factores percepción personal y actuación docente expliquen al desempeño estudiantil. En el proceso de modificación del modelo, se verificó el buen ajuste del modelo de ecuación estructural modificado el cuál incorporó en el modelo propuesto la relación el rendimiento pasado explica a la percepción personal, y se volvió a confirmar los resultados obtenidos anteriormente, así como la significancia de esta nueva relación. Con el modelo modificado se obtuvieron mejores valores de los indicadores de ajuste que con el modelo propuesto. Tomando como base este estudio, en uno posterior se puede incluir en el modelo propuesto otro factor como la habilidad del alumno, para evaluar la significancia de este factor en la explicación del desempeño estudiantil.

\section{Conflictos de intereses}

Los autores firmantes del presente trabajo de investigación declaran no tener ningún potencial conflicto de interés personal o económico con otras personas $\mathrm{u}$ organizaciones que puedan influir indebidamente con el presente manuscrito. 


\section{Contribuciones de los autores}

Preparación y ejecución: RS, FR; Desarrollo de la metodología: RS, FR; Concepción y diseño: RS, FR; Edición del artículo: RS, FR; Supervisión del estudio: RS, FR.

\section{LITERATURA CITADA}

- Aldás, J., \& Uriel, E. (2017). Análisis multivariante aplicado con R. 2da Edición. Ediciones Paraninfo, S.A., Madrid, España, 531 p.

- Cruz, M. (2016). Factores que influyen en el rendimiento académico del estudiante. Escenarios: empresa y territorio, 5(5), 113.

- Flores, Y., Lugo, G., Ramirez, T., Brito, F., \& Crespo, V. (2019). Factores asociados al rendimiento académico de los estudiantes en la Facultad de Odontología de la Universidad Central de Venezuela diseño y validación de un cuestionario, Acta Odontológica Venezolana, $57(2), 6$.

- Garbanzo, G. (2007). Factores asociados al rendimiento académico en estudiantes universitarios, una reflexión desde la calidad de la educación superior pública. Educación, 31(1), 58
- Gargallo, B., Garfella, P., Sánchez, F., Ros, C., \& Serra, B. (2009). La influencia del autoconcepto en el rendimiento académico en estudiantes universitarios, REOP, 20(1), 18, 24.

- Guzmán, M. (2012). Modelos predictivos y explicativos del rendimiento académico universitario: caso de una institución privada en México. Memoria para optar el grado de Doctor. Universidad Complutense de Madrid, Madrid, España. 58, 118-119 p.

- Hair, J., Anderson, R., Tatham, R., \& Black, W. (1999). Análisis Multivariante. 5ta Edición. Prentice Hall Iberia, S.R.L., Madrid, España, 620-683 p.

- Muelas, A. (2013). Influencia de la variable de personalidad en el rendimiento académico de los estudiantes cuando finalizan la Educación Secundaria Obligatoria (ESO) y comienzan Bachillerato, Historia y Comunicación Social, 18 (Esp.), 123.

- Schumacker, R., \& Lomax, R. (2016). A Beginner's Guide to Structural Equation Modeling. 4ta edición. Taylor \& Francis Group. New York, Estados Unidos. 108-109 p.

- Véliz, C. (2016). Análisis multivariante.1ra Edición. Cengage Learning. Lima, Perú, 173$174 \mathrm{p}$. 